\title{
PRELIMINARY STUDY OF ELECTROPHORETIC DEPOSITION OF VERTICALLY ALIGNED MWCNT ON METALLIC ELECTRODE
}

\author{
SIAVASH KHABAZIAN \\ Nanomaterials Group, Department of Materials Science and Engineering, Tarbiat Modares University, \\ Tehran, P.O.Box:14115-143, Iran \\ Khabazian@modares.ac.ir \\ SOHRAB SANJABI ${ }^{\mathrm{a}}$ \\ Nanomaterials Group, Department of Materials Science and Engineering, Tarbiat Modares University, \\ Tehran, P.O.Box:14115-143, Iran \\ Sanjabi@modares.ac.ir
}

\begin{abstract}
Multi-walled carbon nanotubes films formed randomly aligned laterally by electrophoresis. Multi-walled carbon nanotubes with lengths of about $10 \mu$ was shortened and functionalized by a mixture of sulfuric and nitric acid. The functional groups on carbon nanotubes were elaborated by FT-IR. Chemically shortened MWCNTs disperse in organic and aqueous solvent and deposited on electrode vertically-aligned by applying a constant DC electric field. The alignment of MWCNTs was observed by scanning electron microscopy. It also the effect of various substrates on alignment of multi-walled carbon nanotubes was investigated.
\end{abstract}

Keywords: Alignment; Carbon Nanotube Growth; Electrophoresis.

\section{Introduction}

Since their discovery in $1991^{1}$, carbon nanotubes (CNTs) have attracted great attention in various potential applications involving nanoelectronic devices ${ }^{2}$, probe tips for scanning probe microscopy ${ }^{3}$, reinforcing materials ${ }^{4,5}$, field emission ${ }^{6}$, etc. One of the challenges is to tackle the problem of manipulating CNTs, individually or collectively on the support surface at room temperature, to produce a particular arrangement for a given application. One very promising technique being developed for manipulating of CNTs is electrophoretic deposition (EPD). EPD offers advantages of low cost, process simplicity, uniformity of deposits and so on. Carbon nanotube films which form by electrophoretic deposition are uniform but CNTs are laterally deposited on the substrate and they are rarely aligned ${ }^{7,8}$. Applied fields are known to polarize individual CNTs, generating a

\footnotetext{
${ }^{\text {a }}$ Corresponding author
} 
dipole moment which tries to orient CNTs parallel to the applied electric field ${ }^{7}$. A singlewalled carbon nanotube consists of one cylindrical graphene sheet and has a diameter from 1 to $5 \mathrm{~nm}$, whereas a multi-walled carbon nanotube is an arrangement of coaxial tubes of graphene sheets forming a tube like structure with a diameter ranging from 2.5 $\mathrm{nm}$ to several hundred $\mathrm{nm}$. The effect of length and diameter and electrical conductivity on polarization in CNTs was studied by Farajian et al ${ }^{9}$ and it was revealed that lower electric fields were needed for polarization of lower diameter and longer CNTs whereas the critical electric field for polarization of CNTs reduced when conductivity of CNTs was increased. Many researchers investigated the alignment of carbon nanotubes on metallic electrodes ${ }^{10,11}$ by using an AC or DC electric fields in which CNTs were aligned between microelectrodes or inter-digitated electrode array. In both cases, CNTs were adhered weakly on the substrate and when the electric field became zero CNTs lost their orientation. Tomuro et al produced highly densified single walled carbon nanotube films formed on a broad area of a flat electrode by electrophoretic deposition ${ }^{9}$. These have been randomly aligned laterally and also compressed by a strong electric field, resulting in almost flat films, whose random orientation limits their technological applications especially in field emission devices. In the present paper, we investigated the alignment of multi walled CNTs between metallic electrodes by electrophoretic deposition and discuss the influence of electric field and substrate on the alignment behavior of multi-walled carbon nanotubes.

\section{Experimental}

\subsection{CNTs preparation}

Multi-walled CNTs used in this study were catalytically grown with 40-60 nm diameter and 1-2 $\mu \mathrm{m}$ length, supplied from Nanochem Company. For functionalization and shortening of CNTs, 1 gram of as purchased CNTs added to $80 \mathrm{ml}$ of acid mixture of sulfuric and nitric in 3:1 ratio in a round-bottomed flask, and heated up to $60{ }^{\circ} \mathrm{C}$ for 4 hours in which it was stirred by a magnetic stirrer at a constant speed of 300rpm. After complement of reactions, the mixture was washed with distilled water on a PTFE membrane $(0.2 \mu \mathrm{m}$ pore size $)$ until the $\mathrm{pH}$ reaches to 7 , followed by drying in an oven at $40^{\circ} \mathrm{C}$ for 48 hours. Both untreated and oxidized materials were characterized by scanning electron microscopy. For this purpose CNTs were dispersed in acetone and the mixture was pipetted on a glassy lam. A statistical evaluation of CNTs size distribution was performed from number of SEM images. Resulted showed the functionalized groups characterized using infrared spectroscopy (FT-IR).

\subsection{CNTs electrophoretic deposition}

Electrophoretic deposition was carried out under different conditions, including field strength and different kinds of substrate including copper, electroplated nickel on a copper substrate, gold sputtered on a copper substrate and finally stainless steel 316L which in each experiment both of electrodes were made of one of the materials described 
above separated by a Teflon spacer with a gap size of $8 \mathrm{~mm}$ and the time of deposition was 5 minutes. In all EPD experiments dimension of electrodes were $15 \times 15 \times 0.25 \mathrm{~mm}^{3}$ and before performing the experiments, the electrodes were degreased with acetone followed by distilled water. The solution for EPD was prepared by dispersing in Dimethylformamide (DMF) and water at concentration of $0.01 \mathrm{mg} / \mathrm{ml}$. To produce a well dispersed stable CNT suspension, an ultrasonic homogenizer (Hielscher, 400 UL) was subjected to the suspension for $30 \mathrm{~min}$ followed by centrifugation of suspension for $5 \mathrm{~min}$ at $5000 \mathrm{rpm}$. The upper solution was used for the electrophoretic deposition and the residues were removed. After deposition electrodes were rinsed in ethanol and dried in air at room temperature. Scanning electron microscope was used to characterize coatings. The indication for suitable adherence of coatings was that when CNT films are rinsed in water they do not alter.

\section{Results and discussion}

CNTs produced by chemical vapor deposition inherently have many defects on their sidewalls and also five or seven-membered rings on their caps which are less stable than carbon atom hexagons ${ }^{12}$. It is shown that CNTs refluxed in boiling $\mathrm{HNO}_{3}$ for $24 \mathrm{~h}$ just lose their cap without any reduction in length ${ }^{13}$, but in the presence of $\mathrm{H}_{2} \mathrm{SO}_{4}$ which increase the corrosion ability, the length of CNTs is reduced proportional to the time of oxidation, temperature and concentration of acidic mixtures ${ }^{14}$. Many literatures shown that acidic treatment produced functional groups such as carboxylic, phenolic and lactonic groups, predominantly situated on the position of open ends and less on their sidewalls. These functional groups result in electrostatic repulsion among the nanotubes and lead to a dramatic increase in stability of the colloidal suspension ${ }^{15}$, ${ }^{16}$ Fig. 1a \& b present SEM micrographs of as purchased and acid treated MWCNTs respectively. Nanotube length distribution histogram corresponding to the respective image in Fig. 1 is shown in Fig. 2. The length of curved MWCNTs was measured with a flexible ruler. Raw MWCNTs are severely bent and are too long (see Fig. 1. a). Shortening process for 4 hours produce straighter and shorter CNTs which are more suitable for EPD. The mean length of oxidized MWCNTs is $470 \mathrm{~nm}$.
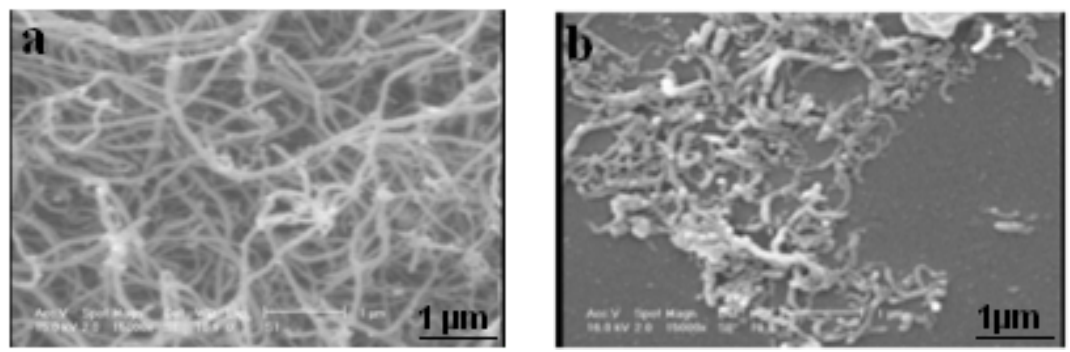

Fig. 1. SEM images of (a) raw MWCNTs (b) shortened MWCNTs 


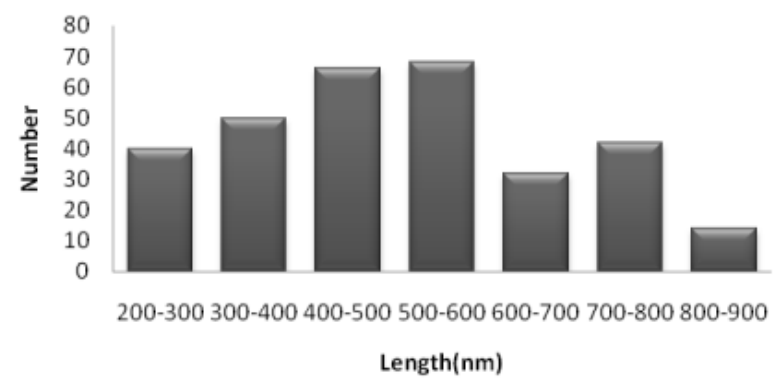

Fig. 2. Histogram of the length of treated MWCNTs

Fig.3 shows the FT-IR spectra of the raw and treated MWCNTs. The as-received MWCNTs showed one peak at around $1700 \mathrm{~cm}^{-1}$ assigned to the oscillation of carboxylic groups which shifts to slightly higher wave numbers after acid treatments. It should be noticed that the as-received MWCNTs were purified by acidic treatment which can introduced carboxylic site on as received MWCNTs, this band displaced to higher frequencies by shortening treatment with the acidic mixtures. The peak at $1540 \mathrm{~cm}^{-1}$ can be associated with the stretching of carbon nanotubes backbone ${ }^{17}$. Both peaks remain present in the spectra independent of the surface chemical treatment, whilst chemical treatment with the acid mixture introduces additional peaks in the spectra of the samples treated. One small peak appearing at $1400 \mathrm{~cm}^{-1}$ is possibly related with $\mathrm{O}-\mathrm{H}$ bending deformation in carboxylic acid groups which increased strength of the signal at 1200 $\mathrm{cm}^{-1}$ may be attributed to $\mathrm{C}-\mathrm{O}$ stretching in the same functionalities. In addition, a small peak appears at $1040 \mathrm{~cm}^{-1}$, which can be associated with either $\mathrm{R}-\mathrm{O}-\mathrm{R}$ functionalities, for $\mathrm{H}_{2} \mathrm{SO}_{4} / \mathrm{HNO}_{3}$ treatment.

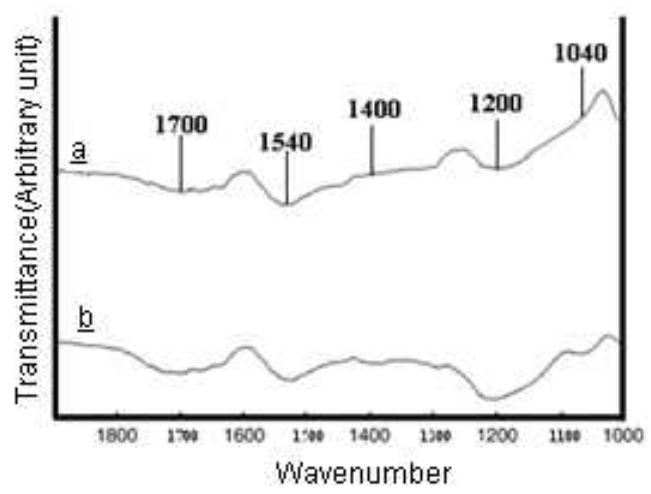

Fig. 3. FT-IR spectra of treated MWCNTs (a) raw MWCNTs (b) treated MWCNTs 
The migration direction of CNTs during EPD is controlled by surface charge and in this case CNTs moved toward the positive electrodes and it seems that oxidized CNTs have negative charges on their surface which confirmed by the others ${ }^{18}$. Electrophoretically deposited CNTs films was formed from water suspension by applying different electric field strength. When distance between electrodes was above $2 \mathrm{~mm}$ and strength of electric fields was above $70 \mathrm{~V} / \mathrm{cm}$, hydrogen evolution on the cathode result in nonuniform coatings. For overcoming this problem we chose $2 \mathrm{~mm}$ distance in which hydrogen evolution was very low. Fig.3 shows the evolution of the current profile with time for to applied voltage. As we can see, the current intensity immediately decreased after applying voltage and then reaches to the steady state as deposition progresses. Decrease in current at initial stage can contribute to the sudden increase of concentration of charged CNTs near electrode and then a concentration potential is produced near positive electrode opposing to the applied electric field and consequently the current is reduced.

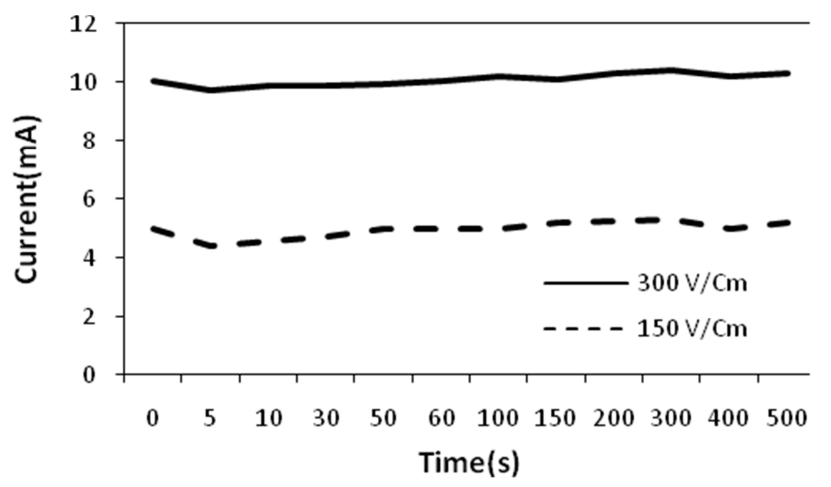

Fig. 4. Current versus times of EPD at different field strength, distance between two electrodes is $2 \mathrm{~mm}$

When electric field was $75 \mathrm{~V} / \mathrm{cm}$ a non-uniform film was deposited and adherence of coating on substrate was very low. By increasing the strength of electric field to $150 \mathrm{~V} / \mathrm{cm}$ (see Fig.5 a) CNT film became uniform and CNTs were deposited laterally with good adhesion and there was much porosity between CNTs which contributes to the weakness of electric field. When electric field was $300 \mathrm{~V} / \mathrm{cm}$ a very compact and uniform film (see Fig.5 b) was formed on the substrate and the porosity in the film decreases as a result of an increase in the field strength. It must be noted that by increasing electric field strenght CNTs are expected to be polarized and therefore align in the direction of electric field as demonstrated by Johann Cho et $\mathrm{al}^{7}$, but in this case we believe that when the distance of electrode keeps $2 \mathrm{~mm}$, the frings of the electric field ${ }^{19}$ become nonuniform especially in the narrow gap we used. 

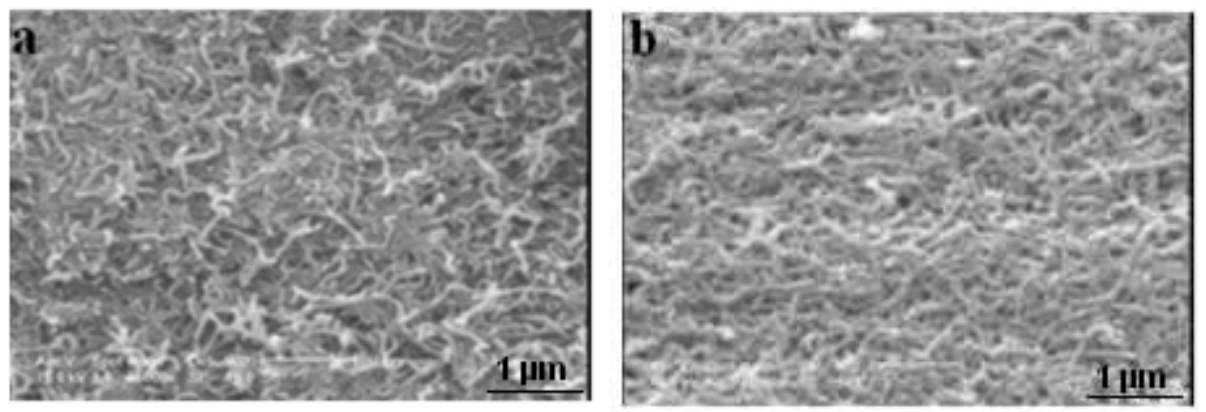

Fig. 5. SEM images of CNTs films which formed by applying (a) $150 \mathrm{~V} / \mathrm{cm}$ and (b) $300 \mathrm{~V} / \mathrm{cm}$

We also used different substrates introduced in the experimental section. Table 1 shows the results for different substrates.

Table 1. Effects of different substrates on the electrophoretic deposition of MWCNTs.

\begin{tabular}{ccc}
\hline Electrode & Adherence & uniformity \\
\hline $\begin{array}{c}\text { Cold rolled Copper } \\
\text { Nickel electroplated on } \\
\text { copper }\end{array}$ & No adherence & -oo-------- \\
$\begin{array}{c}\text { Gold sputtered on } \\
\text { copper }\end{array}$ & Weak & $\begin{array}{c}\text { Large agglomerate which distribute } \\
\text { on the surface }\end{array}$ \\
\hline
\end{tabular}

It has been shown that ${ }^{20}$ the magnitude of polarization in a sphere-shaped particle with radius $a$, constant electric conductivity $\sigma_{\mathrm{p}}$, suspended in a fluid of conductivity $\sigma_{\mathrm{c}}$ and dielectric constant $\varepsilon_{\mathrm{c}}$, in the presence of a DC electric field with strength of $E_{0}$ is equal to $p_{\text {cond }}$ as:

$$
p_{\text {cond }}=4 \pi \varepsilon_{0} \varepsilon_{c} a^{3} \beta_{\text {cond }} E_{0}
$$

Where $\varepsilon_{0}$ is the permittivity of space $\left(\varepsilon_{o}=8.85 \times 10^{-12} C^{2} J^{1} m^{-1}\right)$ and $\beta_{\text {cond }}$ defined according to:

$$
\beta_{\text {cond }}=\frac{\sigma_{p}-\sigma_{c}}{\sigma_{p}+2 \sigma_{c}}
$$

It shows that the polarisation of particle is proportional to the strength of external applied electric field and the degree of mismatch between the particle and carrier fluid conductivities. We chose DMF as a solvent because of high dielectric constant and also to overcome the problem of hydrogen revolution, and to be able to use higher electric fields. MWCNTs are conductive and as shown in Eq. (1), the difference between conductivity of particle and solvent, and the driving force for alignment are increased. 
SEM image of CNT coating deposited on copper at a constant gap of $8 \mathrm{~mm}$ between electrodes at $350 \mathrm{~V} / \mathrm{cm}$ is shown in Fig. 6.

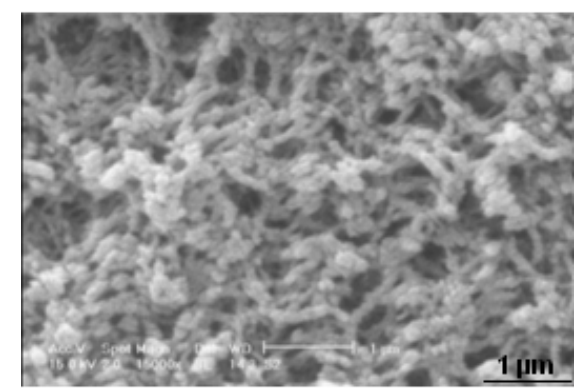

Fig. 6. SEM image of CNTs film which formed on a copper substrate

CNTs are partially aligned to the direction of electric field (see Fig. 6), by applying stronger electric field above $350 \mathrm{~V} / \mathrm{cm}$, in the initial stage of EPD some CNTs coagulated and deposited at the substrate which have low adherence and some other precipitated at the bottom of EPD cell, below of $350 \mathrm{~V} / \mathrm{cm}$ CNTs does not adhere on the electric field because of the lack of suitable electric field. When another substrate was used in our experiment, CNTs doesn't adhere on the substrate.

\section{Conclusion}

We investigated the effect of experimental parameters on alignment of MWCNTs by electrophoretic deposition. When water was used as dispersion media MWCNTs didn't deposit uniformly on copper \& gold and nickel. By increasing electric field strength MWCNTs deposited laterally firmly on the stainless steel electrode. By using DMF, MWCNTs were partially aligned in a range of electric field strengths on copper electrodes. For other electrodes, however, MWCNTs had no adherence on substrate.

\section{References}

1. S, Ijima, Nature. 56, 354 (1991).

2. S. Roth, M. Burghard, V. Krstic, K. Liu, J. Muster, G. Philipp, G.T. Kim, J.G. Park,Y.W. Park, Curr. Appl. Phys. 1, 56 (2001).

3. S. Wong, A. Woolley, E. Joselevich, C. Lieber, Chem. Phys. Lett. 306, 219 (1999).

4. P. Ajayan, L. Schalder, C. Giannaris, A. Rubio, Adv. Mater. 12, 750 (2000).

5. Z. Jin, K.P. Pramoda, G. Xu, S.H. Goh, Chem. Phys. Lett. 337, 43 (2001).

6. J. M. Bonard, H. Kind, T. Stockli, L.O. Nilsson, Solid State Electron. 45, 893 (2001).

7. Johan. Cho, Katarrzyna. Konopka, K, Carbon. 47, 58 (2009).

8. H. Zhao, H. Song, Z. Li, G. Yuan, Y.Jin, Appl. Surf. Sci. 251, 242 (2005).

9. A. Farajian, O. Pupysheva, H. K. Schmidt, Phys. Rev. B. 77, 205432 (2008).

10. X.liu, J. L. Spencer, A.B. Kaiser, W. M. Arnold, Curr.Appl.Phys. 4, 125 (2004). 
11. K. Yamamoto, S.Akita, Y.Nakayama, J. Phys. 31, 35 (1998).

12. B.C. Satishkumar, A.Govindaraj, J.Mofokeng, G.N Subbabba, C. N. Rao, J. Phys. B. 29, 4925 (1996).

13. C. Du, J. Yeh, N.pan, J. Mater .Chem. 15, 548 (2005).

14. J. Li, Y. Zhang, Appl. Surf. Sci. 252, 2944 (2006).

15. M. S. P. Shaffer, X. Fan, Carbon. 36, 603 (1998).

16. T.Saito, K.Matsushige, K.Tanaka. Physica B. 323, 280 (2002).

17. M. S. P. Shafeer, X. Fan, A. H. Windle, Carbon. 36, 1612 (1998).

18. Tang, X., C. Wu and H. Conrad, 1995, J. Appl. Phys. 78, 4183 (1995).

19. D.A. Kurnosov, A.S. Baturin, A.S. Bugaev, K.N. Nikolski, R.G. Tchesov, E. P. Sheshin, Appl .Surf. Sci. 251, 242 (2004).

20. H. See, Korea.Aus. Rheo. J. 11, 169 (1999). 